\title{
An advanced service provision framework for reconfigurable mobile networks
}

\section{Spyridon Panagiotakis*, Maria Koutsopoulou, Athanassia Alonistioti, Nikos Houssos, Vangelis Gazis and Lazaros Merakos}

Communication Networks Laboratory, Department of Informatics \& Telecommunications, University of Athens,

Athens 157 84, Greece

E-mail: spanag@di.uoa.gr_E-mail: mkoutsop@di.uoa.gr

E-mail: nancy@di.uoa.gr_E-mail: nhoussos@di.uoa.gr

E-mail: gazis@di.uoa.gr_E-mail: merakos@di.uoa.gr

*Corresponding author

\begin{abstract}
The success of next generation mobile networks is highly dependent on the availability of a plethora of functionality-rich applications, accessible via a variety of network infrastructures and terminals. This can be achieved through the cooperation of various business players in addition to network operators and infrastructure providers. The specification by major standardisation organisations of open APIs for network access by third parties is a significant step in this direction. A critical issue in such architectures is reconfiguration management, which is regarded as the mean to enable new approaches to service provision, customisation and personalisation. In this paper we present our proposal for a generic, open, flexible, service provision framework that supports context-aware service provision and reconfiguration management.
\end{abstract}

Keywords: context awareness; management; open APIs; reconfiguration; service provision.

Reference to this paper should be made as follows: Panagiotakis, S., Koutsopoulou, M., Alonistioti, A., Houssos, N., Gazis, V. and Merakos, L. (2003) 'An advanced service provision framework for reconfigurable mobile networks', Int. J. Mobile Communications, Vol. 1, No. 4, pp.425-438.

Biographical notes: Spyridon Panagiotakis received his BSc and MSc from the Department of Physics of the University of Athens, Greece, in 1997 and 1999, respectively. In 1998 he received a four-year fellowship for postgraduate studies from the National NCSR 'Demokritos'. Since 2000 he has been studying for a $\mathrm{PhD}$ at the Department of Informatics and Telecommunications (University of Athens) working in the area of mobile systems and data networks. His research interests include software reconfigurable mobile systems and networks, service management, location awareness, and the design of open architectures and platforms. He has participated in several national and European projects.

Maria Koutsopoulou received her diploma with distinction in electrical engineering from the Demokritos University of Thrace, Greece, in 1996. In 1997 she received a four-year fellowship for postgraduate studies from the NCSR 'Demokritos'. Since 1999 she has been a PhD candidate at the Department of 


\section{S. Panagiotakis et al.}

Informatics and Telecommunications, University of Athens, working in the area of software reconfigurable radio systems and networks. She has participated in several national and European projects. Her research interests include design of open architectures and platforms, charging, billing and accounting issues for GPRS and UMTS systems, IP networks and reconfigurable networks.

Athanassia Alonistioti has a $\mathrm{BSc}$ and a $\mathrm{PhD}$ degree in informatics and telecommunications (University of Athens). She has worked for seven years at the Institute of Informatics and Telecommunications of NCSR 'Demokritos'. She collaborated for one year as an expert at the Greek regulatory organisation and is currently working as senior researcher and project manager in the Communication Networks Laboratory (University of Athens). She has participated in several national and European projects. She specialises in mobile communications, reconfigurable systems and networks, adaptable service engineering, formal specification and testing of communication protocols and services and communications software engineering.

Nikos Houssos obtained his BSc in informatics (University of Athens) in 1998 and his MSc in telematics (University of Surrey) in 1999. He is a staff member at the Communication Networks Laboratory of the University of Athens, working in the area of mobile service provision. He has participated in several national and European projects. $\mathrm{He}$ is currently pursuing a $\mathrm{PhD}$ at the Department of Informatics and Telecommunications, University of Athens. His research interests relate to flexible services provision in $3 \mathrm{G} / 4 \mathrm{G}$ networks, reconfiguration control platforms, adaptation mechanisms, and advanced business models. He has more than 15 publications in the above areas.

Vangelis Gazis received his BSc and MSc from the Department of Informatics and Telecommunications (University of Athens) in 1995, and 1998, respectively, and his MBA from the Athens University of Economics and Business in 2001. Since 1995, he has been with the research staff of the Communication Networks Laboratory, at the aforementioned department, working in funded research and development projects. In parallel, he worked with a number of established companies in the Greek IT sector. He is currently pursuing a PhD. His research interests include adaptable service provision, reconfigurable systems, quality of service, billing, and business model issues in $3 \mathrm{G} / 4 \mathrm{G}$ networks.

Lazaros Merakos received his diploma in electrical and mechanical engineering (National Technical University of Athens) in 1978, and his MSc and PhD in electrical engineering from the State University of New York in 1981 and 1984, respectively. From 1983 to 1986 he was on the Faculty of Electrical Engineering and Computer Science at the University of Connecticut. From 1986 to 1994 he was on the faculty of the Electrical and Computer Engineering Department at Northeastern University. During the period 1993-1994 he served as Director of the Communications and Digital Signal Processing Research Center at Northeastern University. During the summers of 1990 and 1991 he was a visiting scientist at the IBM T.J. Watson Research Center. In 1994 he joined the faculty of the University of Athens where he is presently a Professor in the Department of Informatics and Telecommunications, and Director of the Communication Networks Laboratory (CNL) and the Networks Operations and Management Center. His research interests are in the design and performance analysis of broadband networks, and wireless/mobile communication systems and services. He has written more than 140 papers in the above areas. Since 1995 he has led the research activities of CNL in the area of mobile communications, in the framework of the Advanced Communication 
Technologies \& Services (ACTS) and Information SocietyTechnologies (IST) programmes funded by the European Union. He is president of the Board of the Greek Universities Network (GUnet) and the Greek Schools Network (EDUnet), and member of the board of the Greek Research Network (GRnet). In 1994 he received the Guanella Award for the Best Paper presented at the International Zurich Seminar on Mobile Communications.

\section{Introduction}

The evolution of computing and communication systems to IP-based platforms and the merging of heterogeneous systems and technologies introduce a new era in service provision to end-users. The success of next generation mobile networks is highly dependent on the availability of a plethora of functionality-rich applications, accessible via a variety of network infrastructures and terminals. This vision can be realised only through the co-operation of various business players (e.g. application developers, content providers), in addition to the network operators (mobile or fixed) and the infrastructure providers [1-5].

In that context, the business models to be adopted in the new era are encompassing the contribution by third party Value Added Service Providers (VASPs), which will be able to offer their Value Added Services (VAS) through the operators' networks under the respective business agreements. The challenge for network operators is to attract and engage third-party application providers while protecting their networks from harmful misuse. The specification by major standardisation organisations (e.g. 3GPP [6], UMTS Forum [7], LIF [8]) of open APIs (such as the OSA [9], Parlay [10] and JAIN [11]) for network access by third parties is a significant step towards this direction. It is envisioned that the support of such standardised APIs could enable network operators to open their networks safely and reduce the complexity involved in delivering applications developed by third parties over public switched and mobile networks $[12,13]$.

However, the road to a dynamic, open environment includes major further challenges like taming the unprecedented complexity of service management that spans multiple domains and the introduction of network and systems that are dynamically adaptive and reconfigurable in order to accommodate service delivery over highly diverse contexts. Supported by this increasingly strong trend to renounce proprietary monolithic approaches and endorse more flexible modular architectures in communication network elements, reconfigurability and adaptability are further critical enablers that come into sight for the introduction of ubiquitous services and applications [1-4].

In general, reconfigurability and adaptability are features that span across the whole service provision domain, from terminals and networks to resources and applications. Although reconfigurability research in its first steps focused primarily on the radio domain (RF processing, A/D conversion, etc) [14], currently a more innovative and forward-looking view is increasingly drawing interest. According to that, reconfigurability encompasses the entire service provision domain, extending from the mobile terminal through the network infrastructure to application and services [2-4]. The intelligence required to seamlessly integrate networks, terminal devices, and applications with different 
capabilities and requirements moves from applications and terminals to the network side in order to orchestrate reconfigurability and allow reconfigurability actions to take place transparently to applications and terminals. The most significant near term impact of reconfigurability is likely to be innovation in the field of service and applications, as a tool to allow rapid and flexible service customisation and new degrees of operator differentiation $[15,16]$.

Although the need for the support of advanced business models, encompassing dynamic provision of diverse VAS and content by VASPs has been addressed in the aforementioned standardisation groups and fora, very limited attempts to provide solutions for an integrated all-encompassing service provision, reconfigurability and adaptability management platform have taken place. In this context, we propose the introduction of an intelligent adaptable service creation, deployment, provision and management framework that facilitates and leverages the effort for adaptable and reconfigurable service introduction and support. We envision that such a framework could act as mediator between VASPs, end-users and network operators, thus simplifying the extremely complex task of service management and provisioning. This middleware encompasses the intelligence and functionality required for adapting and reconfiguring the application, content, and data delivery to context information. Additionally, it efficiently monitors and manages the services, applications and infrastructure, thus lowering the requirements for terminals, networks, devices and services for additional intelligence and self-adaptability [2-4].

In the present paper the enhancement of the dominant 3-tier business models to incorporate such an open framework for the support, management and control of the reconfigurability aspects is introduced (Section 2). The functional aspects adopted and the interactions required among its constituent components to accomplish intelligent, context-aware and reconfigurable VAS registration, deployment and provision, as well as flexible charging for the service consumption, are discussed in Sections 3 and 4. Sections 5 and 6 present the current status and the future plans of our work and Section 7 concludes the paper. The model and architecture introduced have been partially developed under the IST project MOBIVAS [17,18].

\section{Business model}

The introduction of $3 \mathrm{G}$ systems and the convergence of the IP and telecommunications worlds it induces are expected to foster the emergence of hybrid models in service provision, context awareness, and reconfigurability. The transition from a rigid strictly operator-centric network to a dynamic open market should be evolutionary and lead to business models that preserve the positive features of the existing paradigms, while removing some of their limitations.

In such a framework, the typical mobile network operator possesses significant strategic advantages, since it maintains the customer relationship and provides integrated robust authentication mechanisms and rigid security features that establish an important level of trust with its subscribers. Thus, it presents a form of 'bridge' for application and content providers seeking to position themselves in the $3 \mathrm{G}$ market. On the other hand, application and content providers are able to provide an abundance of resources (i.e. applications and content) that can contribute greatly to increased revenues for all involved 
businesses. Moreover, the creation of a dynamic and highly profitable market requires the availability of sophisticated VAS management mechanisms that will enable VAS to be rapidly deployed, quickly and efficiently discovered by end users, and optimally provisioned for end users [19]. Finally, VAS consumption charges must be calculated in flexible ways that make VAS usage more attractive to users. Therefore, we propose a high-level business model that capitalises on the unique value-adding features of each participant. The roles of this model are shown in Figure 1.

Figure 1 Business model for service provision in the forthcoming mobile communications

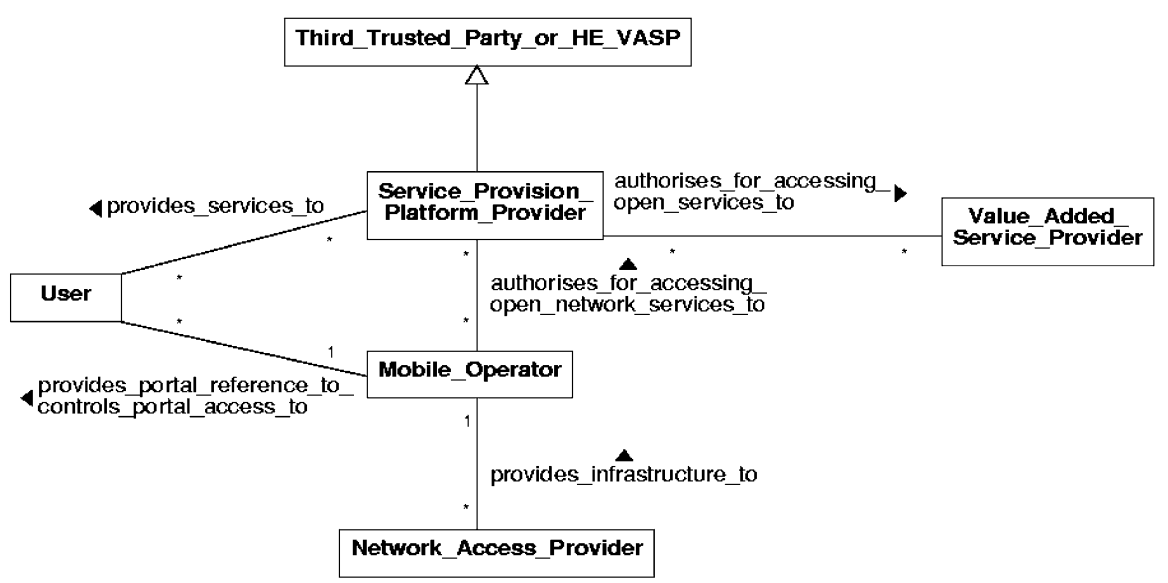

In the proposed business model the following roles are defined:

Mobile user: An entity that is the actual consumer of the available services. The user requests the provision of services and applications from a VASP. The user employs a communication and computing infrastructure contributed the mobile network operator with whom the user maintains a business relationship.

Mobile network operator: The mobile network operator is the entity that provides a network infrastructure and transport medium for authenticated and authorised mobile subscribers to access standardised circuit-switched and packet-switched services (e.g. voice telephony, internet connectivity), as well as VAS and content developed by third parties. It also maintains the customer relationship with the user. The network operator will also typically provide independent third trusted parties and VASPs with access to network functionality, through either standardised open interfaces (e.g. the Open Service Access (OSA) APIs [9]) or a service provision and reconfigurability control middleware.

Reconfigurability control and service provision platform operator: An entity that mediates between VASPs, VAS developers, network operators, and end-users by operating a software platform for VAS management and provision. The platform operator has agreements with network operators that give him access to the underlying network infrastructure, as well as with VASPs for allowing them to provide services and applications to the subscribers of the network operator. 
Value added service provider (VASP): A trusted entity that controls the computational infrastructure utilised to provide applications and services. VAS can range from VoIP and teleconferencing to mobile banking and electronic commerce. VASPs have agreements with platform operators, outsourcing to them the deployment and provision of their VAS on various $3 \mathrm{G}$ networks. Business agreements between VASPs and users are not required; however, they are neither precluded nor ruled out by our model.

In the proposed paradigm, there is not necessarily a one-to-one relationship between roles and real-life entities. For example, a 3G-network operator could also undertake the role of Reconfigurability Control and Service Provision Platform operator.

\section{Flexible service provision and reconfiguration management}

Flexible service provision incorporates the support of VAS introduction, deployment, offering and management. It implies the support of advanced business models (e.g. dynamic service offering by independent VASPs through mobile operators' networks). Moreover, context awareness and personalised service provision (tailored to the user's profile and location, terminal/network profile, and security profile) should be integral parts of any flexible service offering. Incipient architectures for the enabling of flexible service provision comprise the specification of standardised open Application Programming Interfaces (open APIs) (e.g. OSA [9], Parlay [10], JAIN [11] and frameworks [13,20]. Such APIs hide the network heterogeneity that is likely to dominate the forthcoming mobile communications era [21] by providing independence from the underlying network infrastructure to trusted third parties. Therefore, authorised third parties and VASPs can access network services, as well as develop and deploy their services and applications seamlessly, simply by using the standardised execution environment and the associated methods those APIs comprise. However, extensions of the existing standardised APIs with reconfiguration interfaces are required to allow network and device reconfiguration and enable service adaptability and context awareness [4].

We have designed and developed a framework to enable flexible service provision and the support of reconfiguration in order to apply policy-based service management and service provision tailored to context information. We define context as the combination of information relevant to the nearest environment of a user such as the user's location, terminal, network, service and security profiles. The main goal for the design of such a framework is to facilitate the deployment and provision of value added services by independent VASPs that would offer their VAS through an operator's network, as well as to support improved quality in the overall service provision experienced by the end users. Context-aware policies are applied to the underlying network infrastructure through the appropriate API for policy-based management provided by the mobile network operator. Aspects related to service discovery, service registration, service adaptation, terminal/ network reconfiguration, protocol and service downloading, charging, location awareness etc., have been addressed in the design and implementation of the flexible service provision and reconfigurability management framework [22]. This architecture allows the internet to converge seamlessly and transparently with the forthcoming IP-based mobile telecommunications networks. 


\section{A flexible framework for reconfigurable service provision}

To support the demand of future communications networks for efficient and personalised service provision the standard UMTS infrastructure should be supplemented with intelligent components. Figure 2 depicts the general architecture and an example physical placement of the Reconfiguration Control and Service provision Platform (RCSPP) to meet requirements for the era of $3 \mathrm{G}$ and beyond. It is assumed that the independent VASPS offer their Value Added Services using the transport service provided by the underlying UMTS network.

Figure 2 General architecture and example physical placement of the RCSPP framework

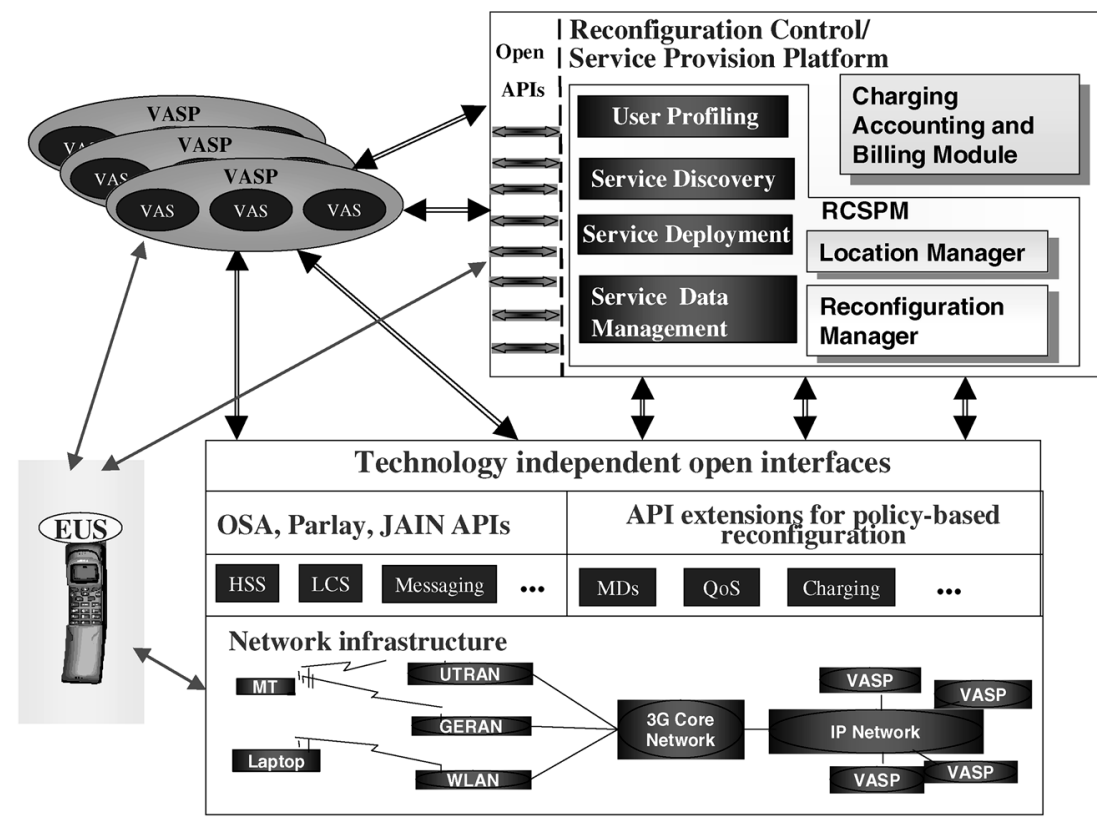

The RCSPP constitutes an integrated distributed software framework for reconfigurable deployment and management of VAS offered to mobile users as well as charging for their usage [2-4]. It can be viewed as an intelligent service middleware that mediates between VASPs and the network operator in order to provide VASPs and end users with advanced context-aware services. In order to accomplish this, the RCSPP takes into account context information such as the location and mobility information for the subscribers, the preferences from their user profiles and the current terminal and network capabilities, to perform reconfiguration actions on the network nodes and end user equipment. More specifically the RCSPP is able to efficiently handle issues related to the management of services, applications and infrastructure, such as the: 
- dynamic VAS deployment

- intelligent and customised VAS discovery

- efficient profile management

- context-awareness in service provision

- efficient event monitoring and handling

- flexible reconfiguration management based on policies

- user notification.

The framework introduces several components involved in service provision namely, the Reconfiguration Control and Service Provision Manager (RCSPM), the Charging Accounting and Billing System (CAB), the Metering Devices (MDs), the User Equipment Software (UES) and various databases. Although business roles may be integrated to one entity (e.g. the mobile operator may undertake the business roles of the RCSPP provider and the network access provider), we see that the business domains can also be physically separated. In more detail:

The Reconfiguration Control and Service Provision Manager (RCSPM) may reside in a third party domain. It coordinates the required procedures for dynamic application deployment, efficient event monitoring and personalised, consistent and reconfigurable discovery, downloading, execution and management of services to mobile users. To accomplish this, it maintains a database with information about the VAS offered by the platform (the VAS DB), as well as user profile data. The VAS are registered in the VAS DB by the corresponding VASP through a web-like open API that facilitates and automates service deployment, making it transparent to the VASPs. This open API provides the third party VASPs with the ability to register new VAS with the platform (providing the RCSPP with the appropriate information about VAS in form of XML-based VAS records), as well as to delete and update existing VAS. The RCSPM then performs all re-configuration actions that are necessary to accommodate the new or updated VAS. These actions comprise the storage of the VAS records in the VAS DB and the configuration/reconfiguration of the Metering Devices for monitoring the IP traffic flows concerning access to the registered VAS. Whenever an end-user requests to discover the available services, the RCSPM filters the VAS DB records, taking into account the applicable terminal device's capabilities, the current user location, the applicable user preferences and his serving network in order to present the user with a VAS listing tailored to his context. The RCSPM retrieves the appropriate context information through the open interfaces provided by the network operator for accessing the associated network servers of the underlying network infrastructure. Each VAS in the service discovery listings is associated with a short description and tariffing information, so that the user may choose VAS based on reduced cost criteria. Figure 3 illustrates the steps required for discovering the available VAS. Following the service discovery phase the end-user is able to select the desired VAS client and to download it to his device $[17,18]$. 
Figure 3 The steps required for discovering services in the RCSPP framework

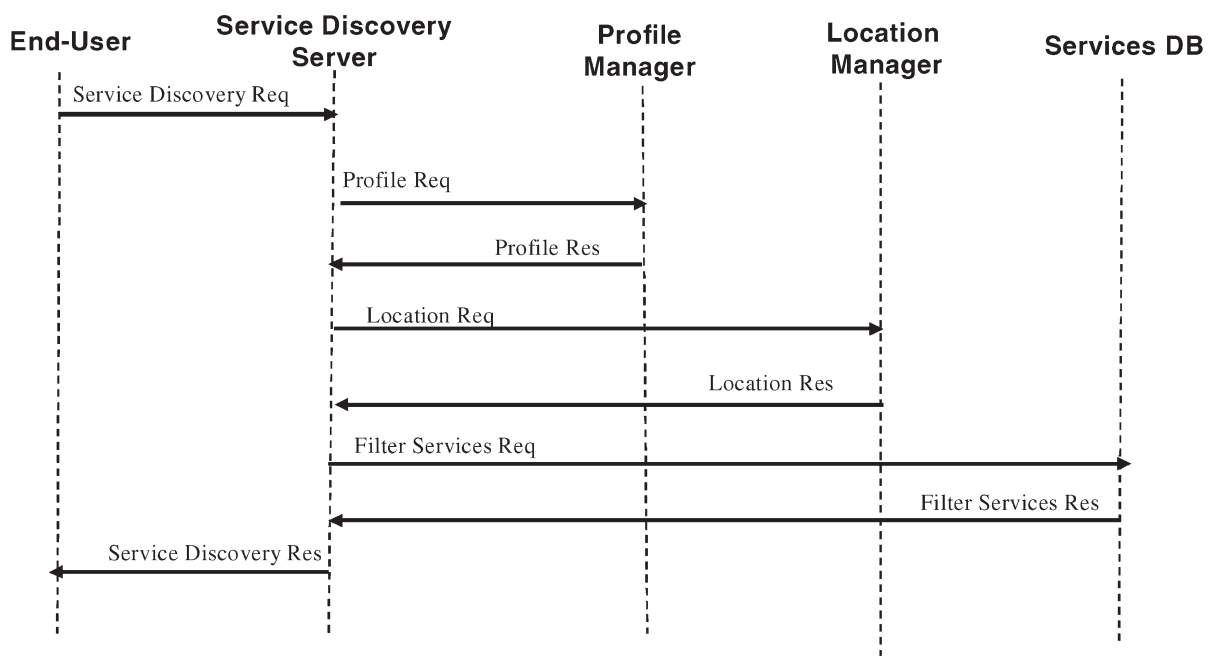

Since the RCSPP may be managed by trusted third parties of the network operator, interactions among the modules of the platform and Basic Network Elements should take place through open network interfaces (APIs). Various industry initiatives, such as OSA [9], PARLAY [10], and JAIN [11], address the introduction of open network interfaces to third party providers. Common to all these architectures is the provision of a Basic Network Management Layer by the mobile operator, which acts as a gateway (mediator) between third parties and provided basic network services [12]. Through such APIs authorised entities are given the ability to retrieve from and store information to the network elements. Industry initiatives have standardised APIs that enable:

- $\quad$ access to the user-specific information kept in the User Profile repository (e.g. the Home Subscriber System (HSS)) of the network operator

- retrieval of location and presence information, as well as registration for specific location- and presence-sensitive event notifications by the Location and Presence Server of the network operator

- the transmission of simple or multimedia messages to the mobile users through the respective Messaging Server

- $\quad$ access to the Charging Service Feature of the network for addition of charges to the subscribers' bills related to the content or products consumption by the respective subscriber.

Although a lot of discussions have taken place in the Parlay Group [10] and the 3GPP OSA Subworking Group [9] for the standardisation of an open API that will enable the policybased management and reconfiguration of network infrastructures, they have not yet matured. Such an API shall grant to the trusted third parties of a network operator open and standardised access to a Policy Decision Point (PDP) [23] for applying reconfiguration policies to the underlying, associated network elements. This API should also enable the policy-based reconfiguration of the network infrastructure by third trusted parties. 
Our Reconfiguration Control and Service Provision Platform assumes the existence of a standardised and policy-based reconfigurable network infrastructure. Therefore we have included in our work a proposed definition and specification of such an open API, providing for policy based management of mobile networks. The policy-based management architecture that we propose assumes that the communication of the involved Policy Decision Point with the associated network elements is built upon common protocols for policy enforcement such as the COPS [23] or COPS-PR [24]. The PDP, thus, should map all incoming calls on the aforementioned open interface to the appropriate COPS/COPS-PR messages for reconfiguring the underlying network elements.

Through this API authorised entities are enabled first to create and then to apply context-aware policies on various components in the mobile network (thus enabling network reconfigurability by third trusted parties), as well as to create events and register themselves for receiving notifications whenever specific events occur. These events could include modifications of the context information kept in various network elements (e.g. the user profiles, the user location, the terminal device capabilities, specific network traffic thresholds) or enhancing the context awareness of registered parties. Hence, by accessing the standard open network interfaces provided by the Basic Network Management Layer, RCSPP is able to build its services based on the network features and functionality offered by the network operator.

The Metering Devices (e.g. the IP meter [25]) are dynamically configured by the RCSPM through policies to process and monitor the traffic over the IP layer as well as to meter data about resource consumption in the network (e.g. transmitted volume) [26]. In the proposed architecture the MDs are located at the edge of the Network Access Provider so that they process all traffic between VASPs and end users. The collected metering data are formatted into appropriate records, the VAS Data Records (VASDRs) and sent to the $\mathrm{CAB}$ for further processing. The VASDRs, apart from calculation of charges, are also used by the $\mathrm{CAB}$ for collecting usage statistics, such as the VAS that a specific user currently executes. The functionality of the MDs is under the supervision of the $M D$ Reconfiguration Manager, which coordinates their policy-based reconfiguration [27]. The reconfiguration of MDs is offered to the RCSPM and other authorised entities (e.g. the Third Party VASPs) through an open API enabling policy-based management [28]. In such a framework the MD Reconfiguration Manager acts as a Policy Decision Point, mediating between authorised entities and MDs to securely grant the Metering Devices' services to the respective parties.

The CAB system [27] is responsible for the overall control of the charging process, providing advanced charging, accounting and billing services to VASPs and end users. To elaborate, the $\mathrm{CAB}$ collects charging information from both the network infrastructure and the MDs, applies the appropriate pricing model, calculates the portions that are due to each business entity, and produces a single itemised bill for each subscriber. Additionally, the $\mathrm{CAB}$ provides advanced charging services through open APIs in order to enable the RCSPM and independent VASPs to apply pricing policies dynamically, to retrieve VAS usage statistics (e.g. the users that currently execute a specific VAS, or the VAS that are currently executed by a specific user), to add content based charges, and to be informed about the current status of their VAS revenues. 
The User Equipment Software (UES) resides in the terminal equipment (e.g. the mobile terminals). It includes functionality related to service downloading management, GUI clients for service discovery and selection, capturing of event notifications as well as service execution management. The UES is able to identify and communicate to the RCSPM information required for customised service provision (e.g. the terminal capabilities).

\section{Current status and future work}

The general philosophy of the framework presented in this paper rises from the approach adopted during the design and implementation of the experimental platform developed within the scope of the IST project MOBIVAS [17,18]. The MOBIVAS platform provides VASPs with a dynamic environment for VAS deployment and offers users a flexible and context aware mean to discover VAS. The official demonstrations and trials of the MOBIVAS were successfully completed in December 2002.

This article presents the necessary extensions to MOBIVAS to constitute an open shared context infrastructure for context-aware VAS deployment and provision that allows total openness and independence of the middleware provider from the network operator, efficient reconfigurability, context information management and advanced location sensitivity.

Most of the functionality described has been designed and verified using the Specification and Description Language (SDL) and a prototype has been implemented in JAVA. The results from the functional simulations show that the architecture concept of the system is valid. Early results from our performance evaluation indicate that the main issue that we have to address is its scalability, since the latency introduced due to the complex procedures it includes to support the required intelligence might be high and affect the total response time of the system.

For instance, Figure 4 illustrates the average end-to-end delay induced by the scenario where 1000 service discovery requests, the generation of which is based on the Poisson distribution, arrive at the Service Discovery Server of the RCSPM. The end-to-end delay describes the latency between Service Discovery Request and Response for the same user. The associated Message Sequence Chart is depicted in Figure 3. The evaluation shows that for fast generation of the Service Discovery Requests (small value of parameter lambda) the average end-to-end delay remains between 4000 and 5000 time units. As the rhythm of the incoming requests slows (high lambda values), the average end-to-end delay is improved. 
Figure 4 End-to-end delay for service discovery in RCSPP

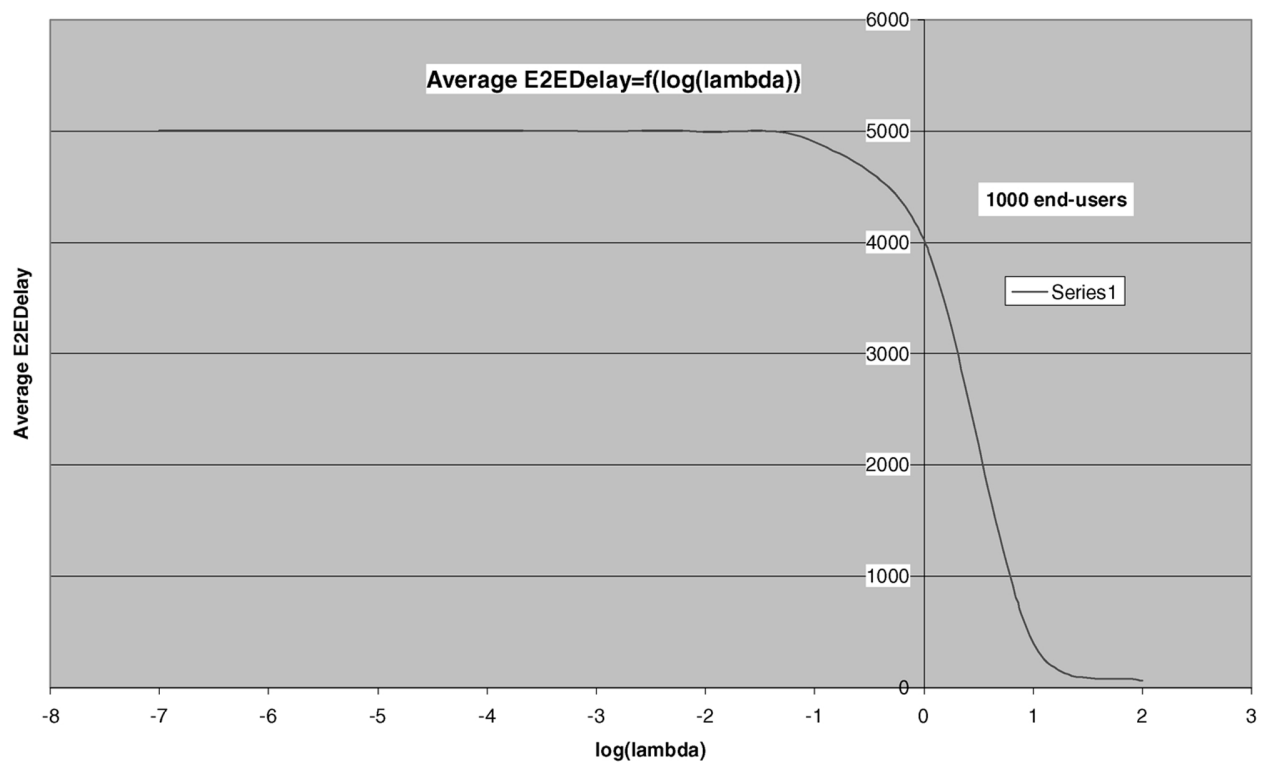

Since the RCSPP is intended to become part of the mobile computing infrastructure, we expect it to scale to a very large numbers of end-users. However, the results show that our system requires the instantiation of additional object components of the RCSPM efficiently to support multiple concurrent users (end-users or registered VAS). The main overhead comes from the time needed to interact with the underlying open network infrastructure to retrieve the context information and apply the reconfiguration policies. Obviously, this is the price we have to pay for introducing the flexible, intelligent middleware we envisage. With additional work on scalability we expect to improve its performance. For example, existing technology for load balancing can be used to scale the system further.

\section{Related work}

In the area of service provisioning, the main approaches are centred on the concepts of OSA [9], Parlay [10], LIF [8], MExE [20], DMTF [13], etc. These approaches focus on the provision of a framework enabling open access to network/terminal functionality and related information. In fact, our platform 'sits' above these technologies, since what we do is use the raw context information provided by their interfaces and technologies to create high level context information and build novel, advanced context aware services.

To date the development of most context-aware applications (e.g. the location-based services) is dominated by the cost of developing a custom context infrastructure for each application. As a result, several context aware platforms, all with almost identical building blocks, have been developed, differentiating themselves mainly by their applications. Our platform attempts to enhance reusability by providing an open interface to applications 
for collecting context information. Furthermore, by incorporating context information interpreters into the infrastructure (such as a spatial database), our platform provides for automatically translating raw context information into high-level context information. VAS developers and providers can access high-level information without needing to invest in their own special equipment (e.g. for location retrieval), or knowing the details of the underlying infrastructure. From the same point of view, most related architectures are focused on collecting context information from local area networks or local sensors and delivering that information to services. Consequently, they tend to cope with adaptable service offering for a user while he moves within a restricted area (e.g. a room, an office, or a school campus). Whereas, our platform collects context information from the underlying mobile networks and offers a wide range of features supporting service adaptability (e.g. for adapting to user location and mobility). Consequently the VAS offered to a user can be adaptable and support a wide range of user mobility. Finally, by registering their VAS with our platform, the VASPs gain access to the subscribers of the mobile network operators.

\section{Conclusions}

This article presents an advanced, open, flexible architecture for adaptable service provision. The platform introduced attempts to substantiate the vision of a reconfigurable network infrastructure that intelligently selects the most appropriate combination of communication protocols, middleware services and application components to efficiently support service requests on a per-user basis. The functional entities introduced, the interactions between them, the open APIs offered and those required for accomplishing reconfigurable service provision were discussed. By mediating between internet application providers and mobile communication operators, this platform will facilitate the seamless convergence of these two worlds and contribute to solving the challenges of the forthcoming era of mobile communications.

\section{References}

1 Pereira, J.M. (2000) 'Re-defining software (defined) radio: re-configurable radio systems and networks', IEICE Transactions on Communications, June.

2 Panagiotakis, S., Houssos, N. and Alonistioti, N. (2001) 'Integrated generic architecture for flexible service provision to mobile users', PIMRC, San Diego, USA.

3 Alonistioti, A., Houssos, N., Panagiotakis, S., Koutsopoulou, M. and Gazis, V. (2002) 'Intelligent architectures enabling flexible service provision and adaptability', WDC 2002, London, UK, 15-17 May.

4 Alonistioti, N., Houssos, N. and Panagiotakis, S. (2001) 'A framework for reconfigurable provisioning of services in mobile networks', ISCTA 2001, Ambleside, Cumbria, UK, July.

5 Gazis, V., Houssos, N., Alonistioti, A. and Merakos, L. (2002) 'Evolving perspectives of 4G mobile communication systems', PIMRC 2002, Lisbon, Portugal, September.

6 3rd Generation Partnership Project (3GPP), http://www.3gpp.org/

7 UMTS Forum, http://www.umts-forum.org/

8 Location Interoperability Forum (LIF), http://www.locationforum.org/ 
9 3GPP TS 29.198: 'Open Service Access (OSA); Application Programming Interface (API); Part 1-12', version 5.0.0, 2002-06.

10 Parlay Group, 'Parlay API Spec. 2.1', July 2000, available from http://www.parlay.org/specs/ index.asp

11 Keijzer, J., Tait, D. and Goedman, R. (2000) 'JAIN: A new approach to services in communication networks', IEEE Communications Magazine, January.

12 Grech, M., McKinney, R., Sharma, S., Stanaway, J., Varney, D. and Vemuri, K. (2000) 'Delivering seamless services in open networks using intelligent service mediation', Bell Labs Technical Journal, July-September.

13 Distributed Management Task Force (DMTF), http://dmtf.org/index.php.

14 Mitola, J. et al. (1999) 'Technical challenges in the globalization of software radio', IEEE Communications Magazine, February.

15 Panagiotakis, S., Koutsopoulou, M., Alonistioti, A. and Kaloxylos, A. (2002) 'Generic framework for the provision of efficient location-based charging over future mobile communication networks', PIMRC 2002, Lisbon, Portugal, September.

16 Houssos, N., Pantazis, S. and Alonistioti, A. (2002) 'Towards adaptability in 3G service provision', IST Mobile Communication Summit 2002, Thessaloniki, Greece, 16-19 June.

17 Houssos, N., Gazis, V., Panagiotakis, S., Gessler, S., Schuelke, A. and Quesnel, S. (2002) 'Value added service management in 3G networks', NOMS 2002, Florence, Italy, 15-19 April.

18 Panagiotakis, S., Houssos, N., and Alonistioti, N. (2001) 'Generic architecture and functionality to support downloadable service provision to mobile users', 3 GIS 2001, Athens, Greece, July.

19 Houssos, N., Gazis, V. and Alonistioti, A. (2002) 'A flexible management architecture for the support of advanced business models in $3 \mathrm{G}$ mobile service provision', 1st International Conference on Mobile Business (M-Business 2002), Athens, Greece, July.

20 3GPP TS 23.057: 'Mobile Station Application Execution Environment (MExE); Functional description; Stage 2', version 5.0.0, 2002-06.

21 Gazis, V., Housos, N., Alonistioti, A. and Merakos, L. (2003) 'Generic system architecture for 4G mobile communications', 57th IEEE Semiannual Vehicular Technology Conference (VTC 2003 - Spring), Jeju, Korea, April.

22 Panagiotakis, S., Koutsopoulou, M. and Alonistioti, A. (2002) 'Advanced location information management scheme for supporting flexible service provisioning in reconfigurable mobile networks', IST Mobile Communication Summit, Thessaloniki, Greece, June.

23 RFC 2748 'Common Open Policy Service protocol (COPS)', Durham, D., Boyle, J., Cohen, R., Herzog, S., Rajan, R. and Sastry, A., available from URL http://www.ietf.org/ rfc/rfc2748.txt

24 RFC 3084 'COPS Usage for Policy Provisioning (COPS-PR)', Chan, K., Seligson, J., Durham, D., Gai, S., McCloghrie, K., Herzog, S., Reichmeyer, F., Yavatkar, R. and Smith, A., available from URL http://www.ietf.org/rfc/ rfc3084.txt

25 Ipmeter White Paper, 2000, http://www.ipmeter.com/doc/ whitepaper/t1.htm.

26 Koutsopoulou, M., Kaloxylos, A. and Alonistioti, A. (2002) 'Charging, accounting and billing as a sophisticated and reconfigurable discrete service for next generation mobile networks', IEEE Semiannual Vehicular Technology Conference (Fall VTC2002), September, Vancouver, Canada.

27 Koutsopoulou, M., Panagiotakis, S., Alonistioti, A. and Kaloxylos, A. (2003) 'An integrated management platform for the support of advanced charging, accounting \& billing schemes in reconfigurable mobile networks', IST Mobile Communication Summit 2003, June, Aveiro, Portugal.

28 Koutsopoulou, M., Alonistioti, N., Gazis, E. and Kaloxylos, A. (2001) 'Adaptive charging accounting and billing system for the support of advanced business models for VAS provision in 3 G systems', PIMRC 2001, San Diego, California, USA, September-October. 